\title{
Seasonal and Temporal Assessment of Surface Water Quality in Saguling Reservoir Indonesia Using Water Quality Index
}

Mariana Marselina ( $\square$ mariana.marselina@yahoo.com )

Institut Teknologi Bandung https://orcid.org/0000-0002-6441-8639

Arwin Sabar

Institut Teknologi Bandung

Nurul Fahimah

Institut Teknologi Bandung

\section{Research}

Keywords: water quality index, inverse distance weight, spatial and temporal assessment

Posted Date: April 2nd, 2020

DOI: https://doi.org/10.21203/rs.3.rs-20562/v1

License: () (i) This work is licensed under a Creative Commons Attribution 4.0 International License. Read Full License 


\section{Abstract}

In recent years, developments in agriculture, industry, and urban activities, especially around rivers and reservoirs have caused significant changes to the quality and quantity of water resources. This includes the Saguling Reservoir, which is located in Citarum basin, Indonesia. A review of previous studies reveals that Water Quality Index (WQI) is efficient for the identification of pollution sources as well as for the understanding of temporal and spatial variations in reservoir water quality. The National Sanitation Foundation - Water Quality Index (NSFWQI), which is a commonly used indicator of surface water quality, is based on turbidity, temperature, phosphate, nitrate, fecal coliform, $\mathrm{pH}, \mathrm{DO}, \mathrm{TS}$, and BOD parameters. Using the results from the correlation matrix, we show that the two water quality parameters that influence the NSFWQI value the most are turbidity and fecal coliform. The average index of NSFWQI was determined to be 48.42 during the dry season, 43.97 during the normal season, and 45.82 during the wet season. A calculation of the WQI classified the water quality in the Saguling Reservoir as "bad" in condition. This study reveals that the strongest and most significant correlation between the concentration parameters and the WQI score is the turbidity concentration fecal coli, which is usable to determine the required parameters for the calculation of WQI with reduced parameters, if needed. This research also conducted nitrate concentration distribution analysis around Saguling Reservoir using the Inverse Distance Weighted method.

\section{Full Text}

This preprint is available for download as a PDF.

\section{Tables}

Table 1. Water quality monitoring locations in the Saguling Reservoir

\begin{tabular}{|c|c|c|c|}
\hline \multirow[t]{2}{*}{ MONITORING STATION } & \multirow[t]{2}{*}{ LOCATION } & \multicolumn{2}{|c|}{ GPS (decimal unit) } \\
\hline & & LS & BT \\
\hline $1 \mathrm{a}$ & Citarum River & $107 \bowtie 32 ' 10,7^{\prime \prime}$ & $06 \llbracket 56^{\prime} 29,8^{\prime \prime}$ \\
\hline $1 b$ & Citarum River Trash Boom Batujajar & $107 \rrbracket 28 ' 35,0 "$ & $06 \llbracket 54^{\prime} 58,9 "$ \\
\hline 2 & Cihaur Cipeundeuy Village & $107 \rrbracket 28 ' 32,3 "$ & $06 \rrbracket 53^{\prime} 13,5^{\prime \prime}$ \\
\hline 3 & Cimerang & $107 \bowtie 277^{\prime} 09,0 "$ & $06 \llbracket 53^{\prime} 13,4^{\prime \prime}$ \\
\hline 4 & Muara Cihaur Kampung Maroko & $107 \rrbracket 25^{\prime} 54,4^{\prime \prime}$ & $06 \llbracket 54 ' 13,0^{\prime \prime}$ \\
\hline 5 & Muara Cipatik & $107 \bowtie 27 ' 25,5^{\prime \prime}$ & $06 \llbracket 56^{\prime} 07,6^{\prime \prime}$ \\
\hline 6 & Muara Ciminyak - floating nets fishing location & $107 \rrbracket 26 ' 03,8 "$ & $06 \varangle 57^{\prime} 14,6 "$ \\
\hline 7 & Muara Cijere & $107 \otimes 24 ' 50,8 "$ & $06 \llbracket 56^{\prime} 14,9^{\prime \prime}$ \\
\hline 8 & Muara Cijambu & $107 \rrbracket 22 ' 22,4^{\prime \prime}$ & $06 \rrbracket 56^{\prime} 00,4^{\prime \prime}$ \\
\hline 9 & Near Intake Structure & $107 \rrbracket 22 ' 26,3^{\prime \prime}$ & $06 \bigotimes 54^{\prime} 54,4^{\prime \prime}$ \\
\hline $10 a$ & Tailrace & $107 \rrbracket 20 ' 57,0 "$ & $06 \llbracket 51^{\prime} 49,8 "$ \\
\hline
\end{tabular}

Table 2. NSFWQI

\begin{tabular}{|lll|}
\hline No & Score & Description \\
\hline 1 & $91 \llbracket 100$ & Excellent water quality \\
\hline 2 & $71 \llbracket 90$ & Good water quality \\
\hline 3 & $51-70$ & Medium or average water quality \\
\hline 4 & $26-50$ & Fair water quality \\
\hline 5 & $0-25$ & Poor water quality \\
\hline
\end{tabular}

Table 3. Weighting factor of water quality parameter for NSFWQI method 


\begin{tabular}{|lllll|}
\hline No & Parameter & Code & Unit & Weighting factor \\
\hline 1 & Temperature & $\mathrm{T}$ & ${ }^{0} \mathrm{C}$ & 0.1 \\
\hline 2 & Dissolved solid & TDS & $\mathrm{mg} / \mathrm{L}$ & 0.07 \\
3 & Turbidity & TUR & $\mathrm{NTU}$ & 0.08 \\
\hline 4 & $\mathrm{Ph}$ & $\mathrm{pH}$ & & 0.11 \\
\hline 5 & Nitrates & $\mathrm{NO}-\mathrm{N}$ & $\mathrm{mg} / \mathrm{L}$ & 0.1 \\
6 & Total Phosphate (TP) & PO4 & $\mathrm{mg} / \mathrm{L}$ & 0.1 \\
\hline 7 & Dissolved oxygen & DO & $\mathrm{mg} / \mathrm{L}$ & 0.17 \\
\hline 8 & Biochemical Oxygen Demand & BOD & $\mathrm{mg} / \mathrm{L}$ & 0.11 \\
\hline 9 & Fecal Coli & FC & Colony $/ 100 \mathrm{~mL}$ & 0.16 \\
\hline
\end{tabular}

Table 4. Water Quality Data of Saguling Reservoir at Normal Year

\begin{tabular}{|c|c|c|c|c|c|c|c|c|c|c|c|c|c|c|c|c|c|c|c|c|c|c|c|}
\hline \multirow{3}{*}{$\begin{array}{l}\text { No } \\
1 \\
1\end{array}$} & \multirow{3}{*}{$\begin{array}{l}\text { Parameter } \\
\text { Location } \\
\text { Nanjung }\end{array}$} & \multicolumn{3}{|c|}{ Temperature } & \multicolumn{3}{|c|}{$\begin{array}{l}\text { Total Dissolved } \\
\text { Solids }\end{array}$} & \multicolumn{3}{|c|}{ Turbidity } & \multicolumn{3}{|l|}{$\mathrm{pH}$} & \multicolumn{3}{|c|}{ Nitrate } & \multicolumn{3}{|c|}{ Phospate } & \multicolumn{3}{|c|}{$\begin{array}{l}\text { Dissolved } \\
\text { Oxygen }\end{array}$} & \multirow{2}{*}{$\bar{n}$} \\
\hline & & ${ }^{\circ} \mathrm{C}$ & & & $\mathrm{mg} / \mathrm{L}$ & & & NTU & & & - & & & $\mathrm{mg} / \mathrm{L}$ & & & $\mathrm{mg} /$ & & & $\mathrm{mg} /$ & & & \\
\hline & & 26,3 & \pm & 1,0 & 241,8 & \pm & 108,4 & 196,3 & \pm & 135,8 & 7,5 & \pm & 0,4 & 2,6 & \pm & 1,5 & 0,5 & \pm & 0,2 & 0,8 & \pm & 1,4 & 4 \\
\hline 2 & Batujajar & 26,8 & \pm & 0,5 & 228,7 & \pm & 93,3 & 87,7 & \pm & 71,0 & 7,4 & \pm & 0,3 & 1,8 & \pm & 0,9 & 0,3 & \pm & 0,1 & 1,0 & \pm & 1,3 & 1 \\
\hline 3 & Cihaur & 27,4 & \pm & 0,4 & 308,2 & \pm & 104,4 & 71,6 & \pm & 59,4 & 7,9 & \pm & 0,4 & 1,9 & \pm & 0,8 & 0,3 & \pm & 0,1 & 1,0 & \pm & 1,4 & 1 \\
\hline 4 & Cimerang & 27,3 & \pm & 0,2 & 252,8 & \pm & 66,7 & 43,6 & \pm & 40,7 & 8,1 & \pm & 1,9 & 2,5 & \pm & 2,0 & 1,3 & \pm & 2,2 & 1,1 & \pm & 1,5 & 1 \\
\hline 5 & $\begin{array}{l}\text { Muara } \\
\text { Cihaur }\end{array}$ & 27,3 & \pm & 0,2 & 203,8 & \pm & 55,8 & 33,6 & \pm & 38,0 & 7,6 & \pm & 0,3 & 1,2 & \pm & 0,4 & 0,4 & \pm & 0,2 & 1,2 & \pm & 1,5 & 1 \\
\hline 6 & $\begin{array}{l}\text { Muara } \\
\text { Cipatik }\end{array}$ & 27,2 & \pm & 0,2 & 123,1 & \pm & 32,4 & 41,9 & \pm & 38,5 & 7,6 & \pm & 0,3 & 1,2 & \pm & 0,2 & 0,4 & \pm & 0,1 & 1,1 & \pm & 1,6 & 1 \\
\hline 7 & $\begin{array}{l}\text { Muara } \\
\text { Ciminyak }\end{array}$ & 27,9 & \pm & 1,3 & 111,1 & \pm & 25,5 & 35,8 & \pm & 31,2 & 7,5 & \pm & 0,2 & 1,4 & \pm & 0,4 & 0,3 & \pm & 0,1 & 1,1 & \pm & 1,4 & 1 \\
\hline 8 & $\begin{array}{l}\text { Muara } \\
\text { Cijere }\end{array}$ & 27,2 & \pm & 0,3 & 143,7 & \pm & 37,5 & 34,3 & \pm & 34,4 & 7,7 & \pm & 0,2 & 1,6 & \pm & 0,6 & 0,3 & \pm & 0,2 & 1,3 & \pm & 1,9 & 1 \\
\hline 9 & $\begin{array}{l}\text { Muara } \\
\text { Cijambu }\end{array}$ & 27,7 & \pm & 1,1 & 155,1 & \pm & 42,8 & 57,2 & \pm & 60,2 & 10,7 & \pm & 6,7 & 1,2 & \pm & 0,6 & 0,3 & \pm & 0,1 & 1,1 & \pm & 1,5 & 1 \\
\hline 10 & Intake & 27,0 & \pm & 0,6 & 161,4 & \pm & 41,3 & 37,4 & \pm & 42,5 & 7,8 & \pm & 0,3 & 1,4 & \pm & 0,6 & 0,3 & \pm & 0,1 & 1,4 & \pm & 1,9 & 1 \\
\hline 11 & Tailrace & 26,7 & \pm & 0,1 & 181,8 & \pm & 0,0 & 32,9 & \pm & 0,0 & 12,0 & \pm & 0,0 & 1,7 & \pm & 0,0 & 0,2 & \pm & 0,0 & 1,0 & \pm & 0,0 & 1 \\
\hline
\end{tabular}

Table 5. Water Quality Data of Saguling Reservoir at Dry Year 


\begin{tabular}{|c|c|c|c|c|c|c|c|c|c|c|c|c|c|c|c|c|c|c|c|c|c|c|c|}
\hline \multirow[t]{2}{*}{ No } & \multirow{2}{*}{$\begin{array}{l}\text { Parameter } \\
\text { Location }\end{array}$} & \multicolumn{3}{|c|}{ Temperature } & \multicolumn{3}{|c|}{$\begin{array}{l}\text { Total Dissolved } \\
\text { Solids }\end{array}$} & \multicolumn{3}{|c|}{ Turbidity } & \multicolumn{3}{|l|}{$\mathrm{pH}$} & \multicolumn{3}{|c|}{ Nitrate } & \multicolumn{3}{|c|}{ Phospate } & \multicolumn{3}{|c|}{$\begin{array}{l}\text { Dissolved } \\
\text { Oxygen }\end{array}$} & $\begin{array}{l}\mathrm{B} \\
\mathrm{O}\end{array}$ \\
\hline & & ${ }^{\circ} \mathrm{C}$ & & & $\mathrm{mg} / \mathrm{L}$ & & & $\mathrm{mg} / \mathrm{L}$ & & & - & & & $\mathrm{mg} /$ & & & $\mathrm{mg}$ & & & $\mathrm{mg} /$ & & & $\mathrm{m}$ \\
\hline 1 & Nanjung & 25,9 & \pm & 0,7 & 320,4 & \pm & 117,0 & 220,9 & \pm & 187,9 & 7,4 & \pm & 0,1 & 3,3 & \pm & 1,1 & 0,4 & \pm & 0,2 & 0,6 & & 1,0 & 5 \\
\hline 2 & Batujajar & 27,5 & \pm & 0,6 & 296,9 & \pm & 52,0 & 50,2 & \pm & 49,2 & 7,6 & \pm & 0,1 & 2,6 & \pm & 0,6 & 0,3 & \pm & 0,1 & 0,9 & & 1,2 & 2 \\
\hline 3 & Cihaur & 27,7 & \pm & 0,5 & 330,5 & \pm & 52,7 & 62,5 & \pm & 45,4 & 8,2 & \pm & 0,3 & 2,4 & \pm & 1,0 & 0,3 & \pm & 0,1 & 0,8 & & 1,1 & 2 \\
\hline 4 & Cimerang & 25,0 & \pm & 5,8 & 266,7 & \pm & 31,6 & 45,6 & \pm & 55,8 & 8,0 & \pm & 0,3 & 2,1 & \pm & 0,7 & 0,2 & \pm & 0,1 & 0,9 & & 1,3 & 1 \\
\hline 5 & $\begin{array}{l}\text { Muara } \\
\text { Cihaur }\end{array}$ & 27,4 & \pm & 0,5 & 250,3 & \pm & 34,8 & 44,0 & \pm & 50,4 & 7,7 & \pm & 0,1 & 2,0 & \pm & 0,5 & 0,3 & \pm & 0,1 & 1,0 & & 1,4 & 1 \\
\hline 6 & $\begin{array}{l}\text { Muara } \\
\text { Cipatik }\end{array}$ & 26,1 & \pm & 3,0 & 139,5 & \pm & 15,9 & 41,9 & \pm & 53,1 & 7,7 & \pm & 0,4 & 1,6 & \pm & 0,4 & 0,3 & \pm & 0,1 & 1,0 & 1 & 1,4 & 1 \\
\hline 7 & $\begin{array}{l}\text { Muara } \\
\text { Ciminyak }\end{array}$ & 27,3 & \pm & 0,4 & 136,9 & \pm & 19,9 & 40,7 & \pm & 52,0 & 7,5 & \pm & 0,1 & 1,6 & \pm & 0,4 & 0,3 & \pm & 0,1 & 0,9 & \pm & 1,3 & 9 \\
\hline 8 & $\begin{array}{l}\text { Muara } \\
\text { Cijere }\end{array}$ & 26,2 & \pm & 3,0 & 162,1 & \pm & 27,0 & 27,8 & \pm & 25,8 & 7,6 & \pm & 0,3 & 1,7 & \pm & 0,4 & 0,3 & \pm & 0 & 1,1 & 1 & 1,5 & 9 \\
\hline 9 & $\begin{array}{l}\text { Muara } \\
\text { Cijambu }\end{array}$ & 27,3 & \pm & 0,3 & 162,2 & \pm & 5,3 & 39,5 & \pm & 53,9 & 7,6 & \pm & 0,2 & 1,4 & \pm & 0,4 & 0,2 & \pm & 0,1 & 1,0 & 1 & 1,4 & 8 \\
\hline 10 & Intake & 26,9 & \pm & 0,2 & 182,5 & \pm & 26,0 & 39,6 & \pm & 39,5 & 11,6 & \pm & 9,1 & 1,6 & \pm & 0,4 & 0,3 & \pm & 0,1 & 1,2 & \pm & 1,7 & 1 \\
\hline 11 & Tailrace & 26,8 & \pm & 0,2 & 209,6 & \pm & 0,0 & 27,6 & \pm & 0,0 & 7,2 & \pm & 0,0 & 1,6 & \pm & 0,0 & 0,2 & \pm & 0,0 & 1,0 & \pm & 0,0 & 9 \\
\hline
\end{tabular}

Table 6. Water Quality Data of Saguling Reservoir at Wet Year

\begin{tabular}{|c|c|c|c|c|c|c|c|c|c|c|c|c|c|c|c|c|c|c|c|c|c|c|c|}
\hline \multirow{3}{*}{$\begin{array}{l}\text { No } \\
1 \\
1\end{array}$} & \multirow{3}{*}{$\begin{array}{l}\text { Parameter } \\
\text { Location } \\
\text { Nanjung }\end{array}$} & \multicolumn{3}{|c|}{ Temperatur } & \multicolumn{3}{|c|}{$\begin{array}{l}\text { Total Dissolved } \\
\text { Solids }\end{array}$} & \multicolumn{3}{|c|}{ Turbidity } & \multicolumn{3}{|l|}{$\mathrm{pH}$} & \multicolumn{3}{|c|}{ Nitrate } & \multicolumn{3}{|c|}{ Phospate } & \multicolumn{3}{|c|}{$\begin{array}{l}\text { Dissolved } \\
\text { Oxygen }\end{array}$} & \multirow{2}{*}{$\frac{\mathrm{E}}{\mathrm{r}}$} \\
\hline & & ${ }^{\circ} \mathrm{C}$ & & & $\mathrm{mg} / \mathrm{L}$ & & & NTU & & & - & & & & & & & $g / L$ & & $\mathrm{mg} /$ & & & \\
\hline & & 25,8 & \pm & 0,4 & 245,5 & \pm & 83,4 & 193,6 & \pm & 101,6 & 7,4 & \pm & 0,2 & 2,5 & \pm & 1,5 & 0,3 & \pm & 0,1 & 1,5 & \pm & 1,4 & 2 \\
\hline 2 & Batujajar & 26,9 & \pm & 0,7 & 213,8 & \pm & 49,6 & 62,9 & \pm & 50,4 & 7,4 & \pm & 0,2 & 1,7 & \pm & 0,8 & 0,3 & \pm & 0,1 & 2,9 & \pm & 3,4 & 1 \\
\hline 3 & Cihaur & 26,1 & \pm & 3,3 & 299,1 & \pm & 77,1 & 56,0 & \pm & 53,0 & 7,4 & \pm & 1,4 & 2,1 & \pm & 1,3 & 0,3 & \pm & 0,2 & 2,2 & \pm & 2,7 & ; \\
\hline 4 & Cimerang & 25,8 & \pm & 3,2 & 240,7 & \pm & 58,8 & 40,1 & \pm & 35,3 & 7,4 & \pm & 0,9 & 1,7 & \pm & 1,0 & 0,2 & \pm & 0,1 & 2,5 & \pm & 3,2 & 1 \\
\hline 5 & $\begin{array}{l}\text { Muara } \\
\text { Cihaur }\end{array}$ & 25,8 & \pm & 3,3 & 200,4 & \pm & 52,4 & 38,7 & \pm & 31,0 & 7,1 & \pm & 0,8 & 2,0 & \pm & 0,8 & 0,2 & \pm & 0,1 & 1,7 & \pm & 1,5 & 1 \\
\hline 6 & $\begin{array}{l}\text { Muara } \\
\text { Cipatik }\end{array}$ & 25,9 & \pm & 3,2 & 123,5 & \pm & 22,0 & 45,3 & \pm & 35,2 & 7,0 & \pm & 0,9 & 1,3 & \pm & 0,7 & 0,3 & \pm & 0,1 & 1,8 & \pm & 1,7 & ؟ \\
\hline 7 & $\begin{array}{l}\text { Muara } \\
\text { Ciminyak }\end{array}$ & 25,9 & \pm & 3,2 & 114,8 & \pm & 23,2 & 40,0 & \pm & 35,2 & 6,9 & \pm & 0,8 & 1,2 & \pm & 0,6 & 0,3 & \pm & 0,1 & 1,7 & \pm & 1,6 & 7 \\
\hline 8 & $\begin{array}{l}\text { Muara } \\
\text { Cijere }\end{array}$ & 25,9 & \pm & 3,3 & 146,1 & \pm & 26,5 & 39,6 & \pm & 34,8 & 7,0 & \pm & 0,9 & 1,5 & \pm & 1,0 & 0,2 & \pm & 0,1 & 1,7 & \pm & 1,5 & 1 \\
\hline 9 & $\begin{array}{l}\text { Muara } \\
\text { Cijambu }\end{array}$ & 25,9 & \pm & 3,5 & 154,6 & \pm & 33,9 & 43,9 & \pm & 40,8 & 7,1 & \pm & 0,8 & 1,4 & \pm & 0,9 & 0,2 & \pm & 0,1 & 1,6 & \pm & 1,5 & c \\
\hline 10 & Intake & 25,6 & \pm & 3,4 & 165,1 & \pm & 39,4 & 39,4 & \pm & 38,8 & 6,8 & \pm & 0,9 & 1,5 & \pm & 1,0 & 0,2 & \pm & 0,1 & 1,9 & \pm & 1,8 & 1 \\
\hline 11 & Tailrace & 25,2 & \pm & 3,2 & 181,5 & \pm & 0,0 & 49,3 & \pm & 0,0 & 6,8 & \pm & 0,0 & 1,4 & \pm & 0,0 & 0,2 & \pm & 0,0 & 1,0 & \pm & 0,0 & 1 \\
\hline
\end{tabular}

\section{Figures}




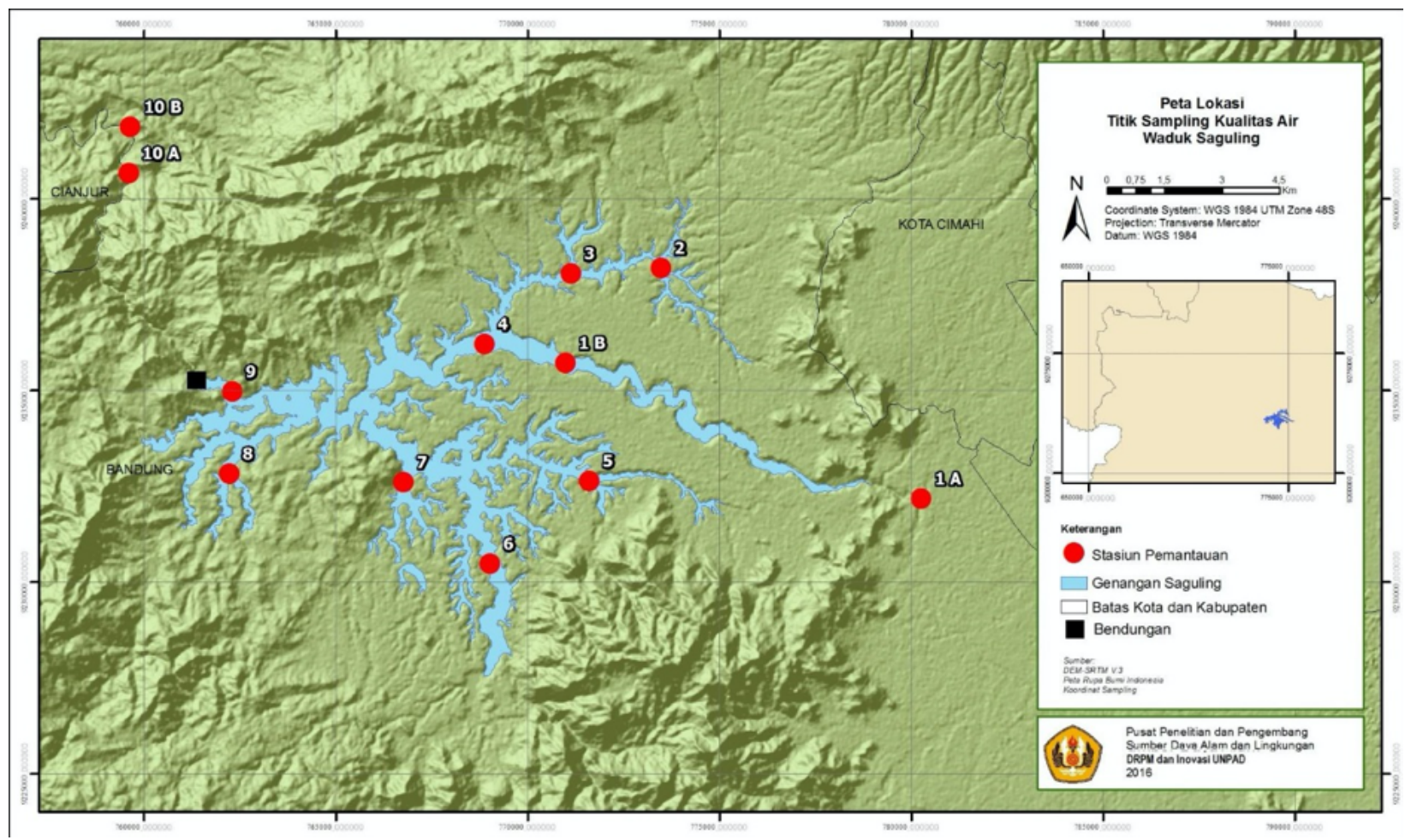

Figure 1

Water quality monitoring locations in the Saguling Reservoir
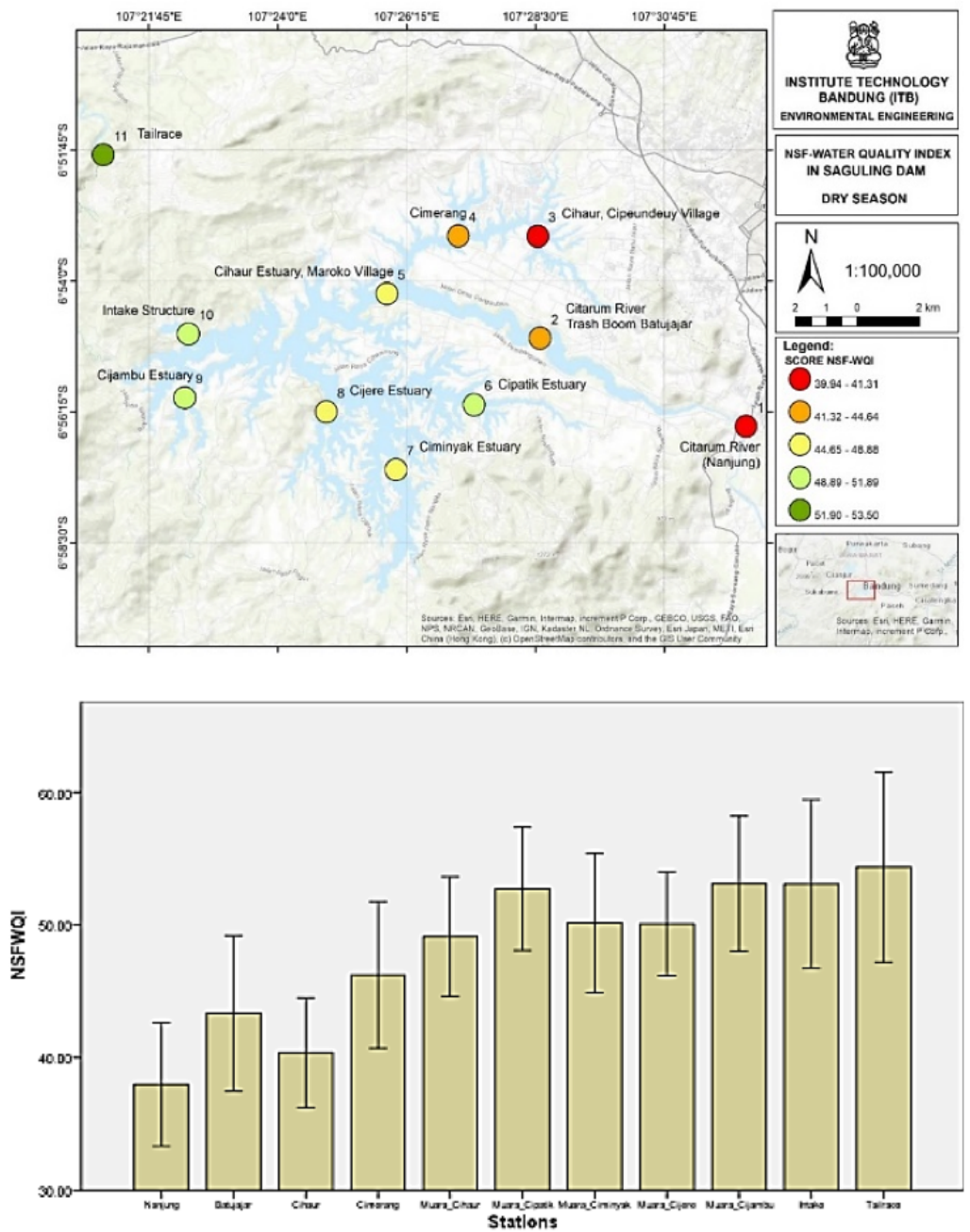
Figure 2

(a). NSFWQI during the Dry Season. (b). NSFWQI value during value the Dry Season for each monitoring location
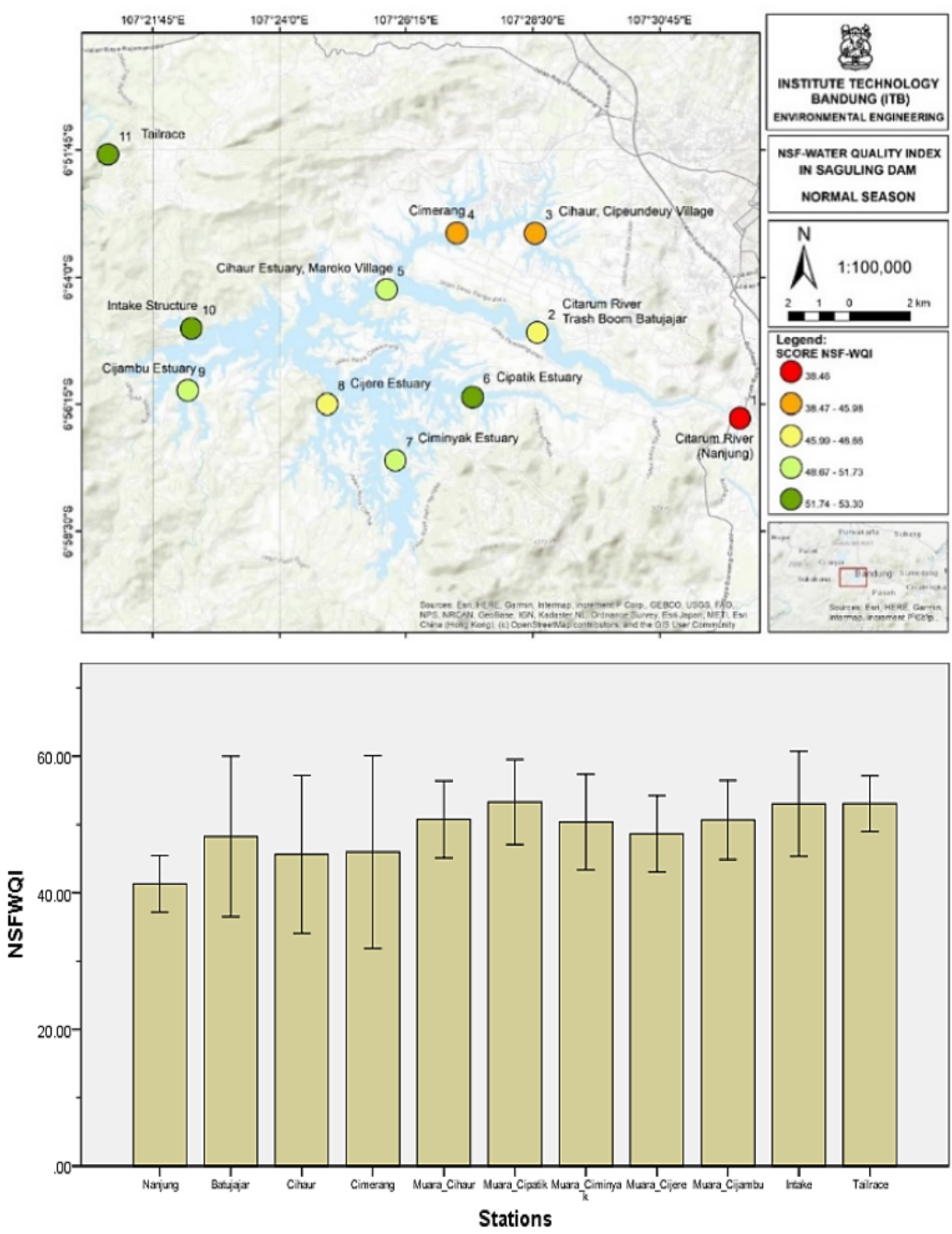

Figure 3

(a). NSFWQI value in the Normal Season. (b). NSFWQI value during the Normal Season for each monitoring location
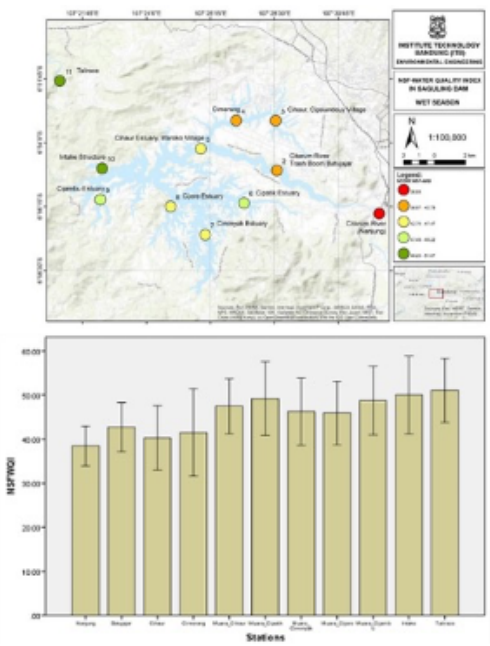

Figure 4

(a). NSFWQI value during the Wet Season. (b). NSFWQI value during the Wet Season for each monitoring location 


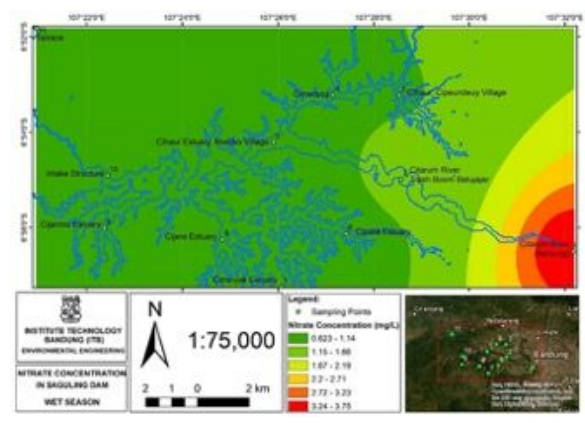

Figure 5

Nitrate distribussion during Wet Year

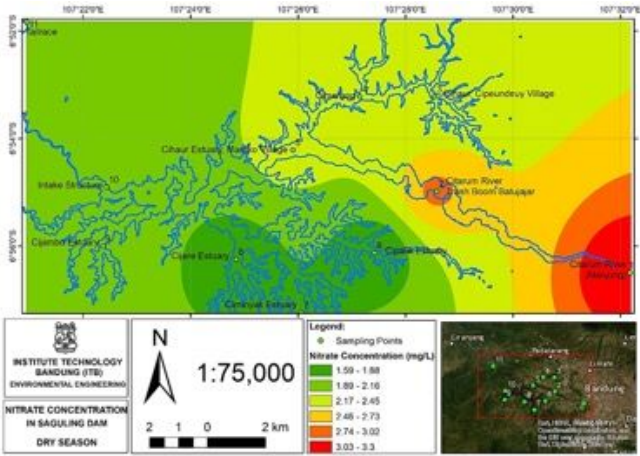

Figure 6

Nitrate distribution at Normal Year

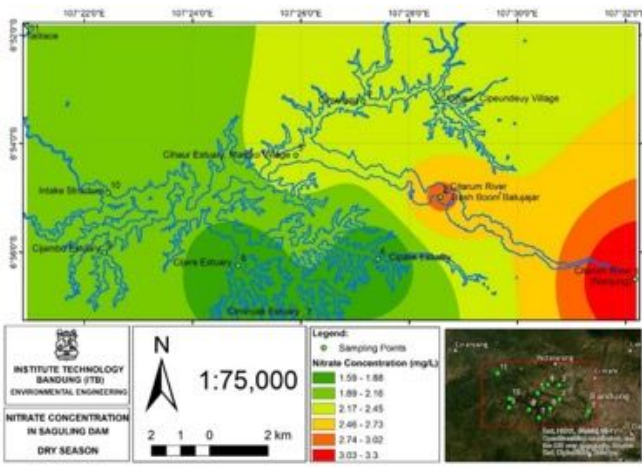

Figure 7

Nitrate distribussion at Dry Year 